\title{
ABNORMALITIES IN THE PULMONARY ARTERIOLES IN SOME CASES OF CORONARY ARTERY DISEASE
}

\author{
BY \\ A. J. THOMAS, W. R. L. JAMES, AND G. M. OWEN \\ From Llandough Hospital (United Cardiff Hospitals), The Institute of Pathology of the Welsh National School of \\ Medicine, and Cardiff Radiotherapy Centre
}

Received August 7, 1961

In 1960 we published a method for assessing the volume of the vascular bed in the peripheral parts of cadaveric lungs (James et al., 1960). We have since used the method in studying the lungs from many cases of chronic cardiac and pulmonary disease. When examining the lungs from patients who had had coronary artery disease we noticed that the severity of the pulmonary arteriolar lesions varied with the clinical history. The purpose of this paper is to demonstrate the relationship between the clinical syndromes and the abnormalities in the pulmonary arteriolar bed.

We were able to study the lungs from two different groups of patients with coronary artery disease. Nine had had prolonged invalidism with many hospital admissions for left ventricular failure or chronic dyspnœa. Eleven others had had no invalidism and had died suddenly apparently while in good health.

\section{METHOD}

At necropsy the main pulmonary artery of one lung was injected with a radiopaque medium containing a known concentration of radio-iodinated serum albumin. It was then fixed and distended by introducing industrial methylated spirit into the main bronchus. The specimen was then radiographed and blocks were taken for histology. Pieces of about $40 \mathrm{ml}$. in volume were then cut from the periphery of the lung. Their volumes were measured by simple displacement. The volume of the vascular bed in each piece was determined by measuring its content of radioactive injected material, using a ring of Geiger counters.

The vascularity of each piece of lung was expressed as a fraction: $\frac{\text { Volume of vascular bed }}{\text { Volume of piece of lung }}$

We measured four pieces from the periphery of each lung and expressed the vascularity of the lung as an average of the four values. We have previously shown that the mean normal value of this fraction was $0 \cdot 2$, the 95 per cent range being 0.1 to 0.3 (James et al., 1960).

\section{RESULTS}

In the eleven specimens from the sudden death group most of our measurements occupied the lower half of the normal range (Fig. 1). In successive cases the values were $0 \cdot 13,0 \cdot 12,0 \cdot 12,0 \cdot 19$, $0 \cdot 17,0 \cdot 20,0 \cdot 13,0 \cdot 14,0 \cdot 13,0 \cdot 10$, and $0 \cdot 12$. Microscopy showed arterioles which were either normal or had minimal degrees of subintimal thickening. The post mortem pulmonary angiograms also showed normal patterns. 


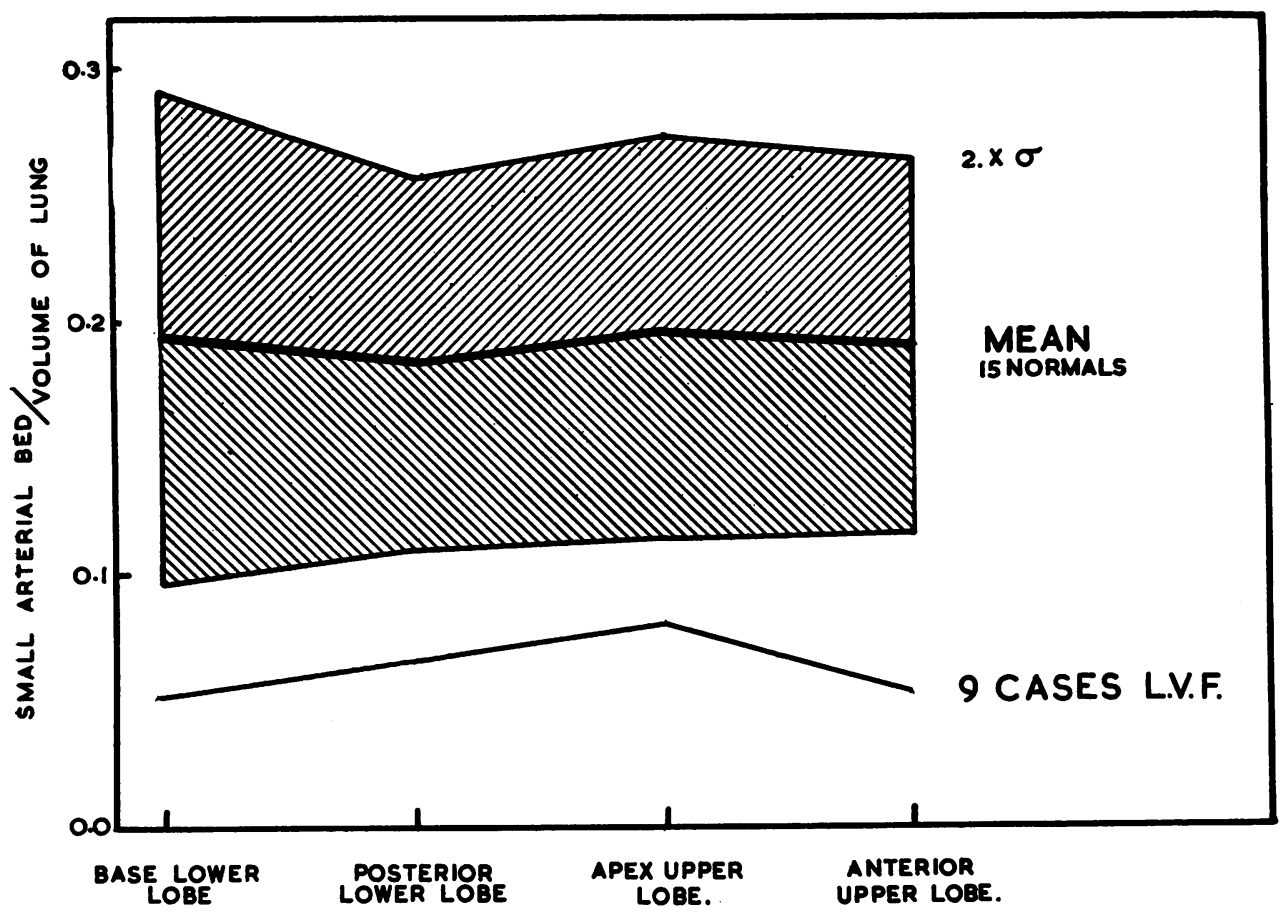

FIG. 1.-The range of the ratio for normal lungs is shown and the mean of the same ratio for the cases of coronary artery disease with left ventricular failure is indicated.

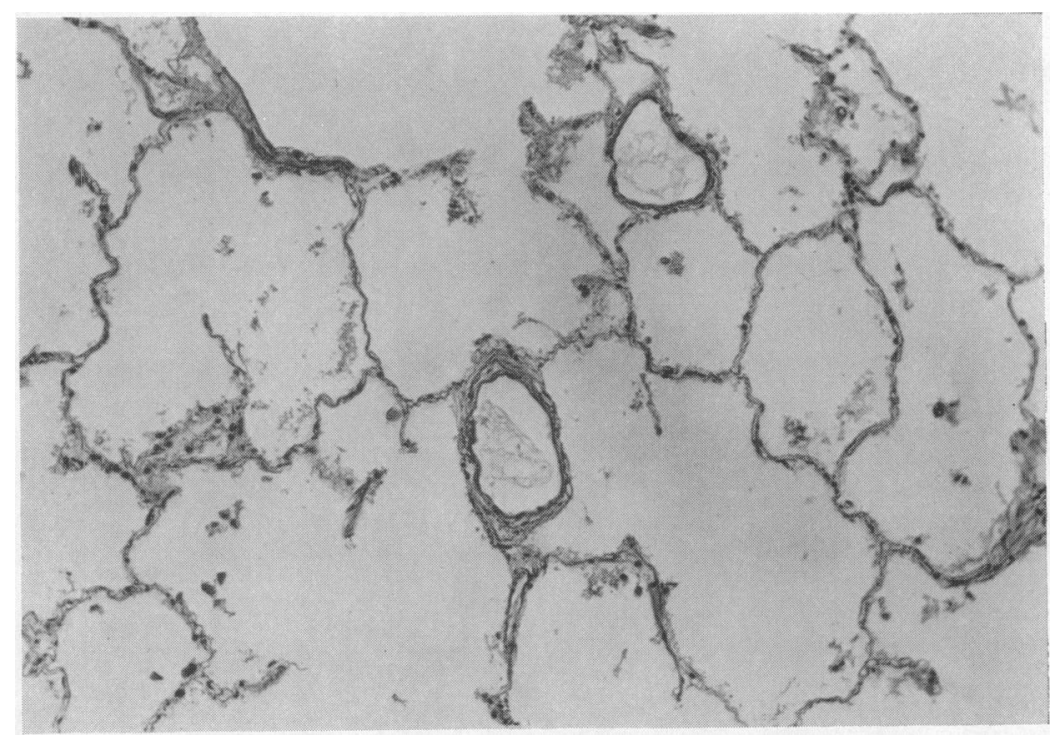

Fig. 2.-Case 25. Two normal pulmonary arterioles from a man of 76 who died suddenly from coronary artery disease without previous symptoms. $(\times 120)$. 
Case 25. A typical example was that of a man of 76 who was considered to be in good health for his age. His sudden death at home was reported to the coroner. Necropsy showed a ruptured recent myocardial infarct. We found the fractional pulmonary vascular bed to be $0 \cdot 17$. The microscopic appearance of the pulmonary arterioles was normal (Fig. 2) and the post mortem pulmonary angiogram showed good filling of the fine vessels (Fig. 3).

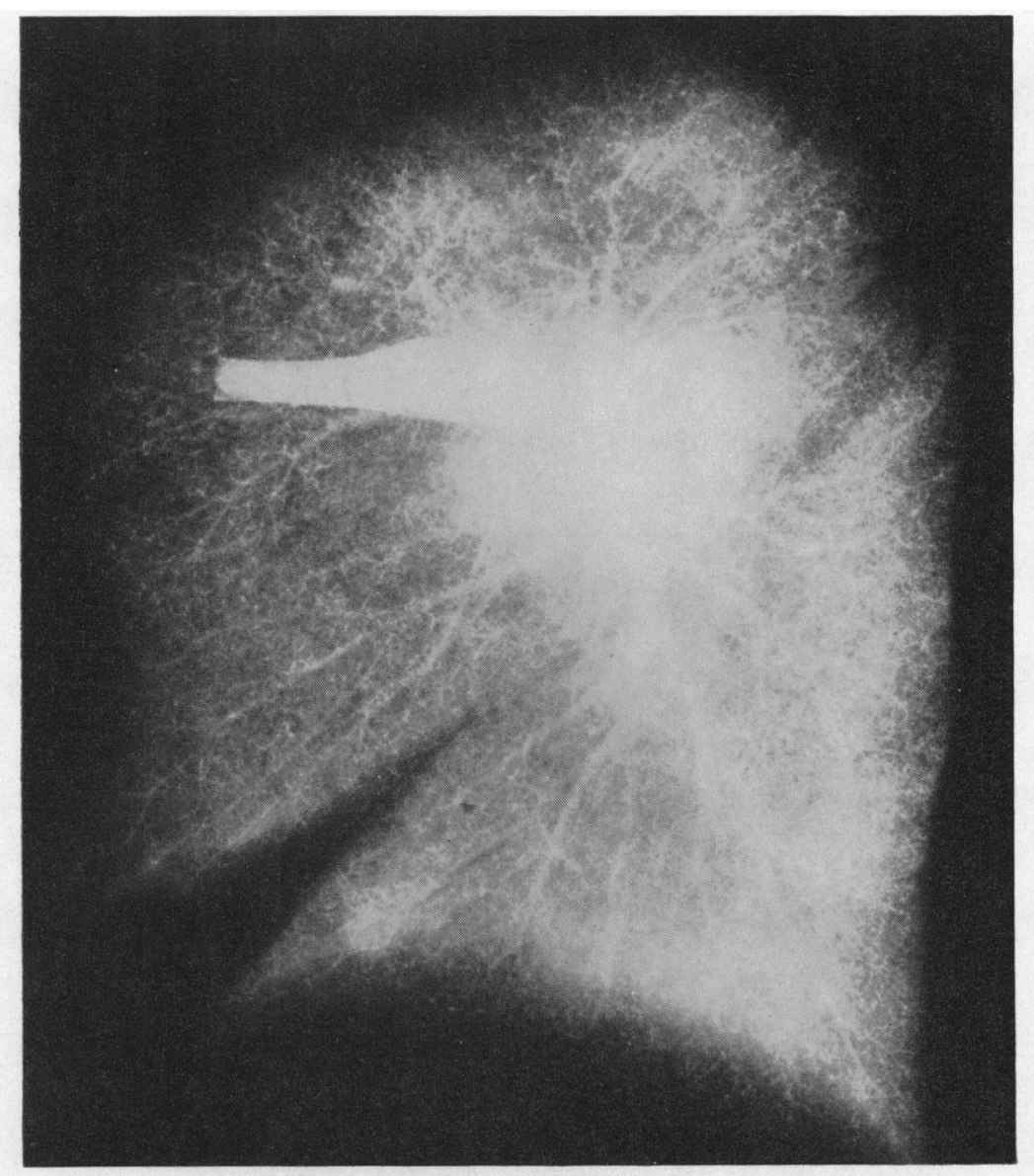

Fig. 3.-Case 25. The pulmonary arteriogram shows normal sized main arteries and good filling of the peripheral small vessels. (About half size.)

In the coronary heart disease group with left ventricular failure or effort dyspnœa we observed considerable differences. In the nine cases our measurements of the vascularity of the lung periphery were $0.09,0.07,0.07,0.07,0.04,0.06,0.04,0.07$, and 0.04 , a considerable reduction below the lowest limit of normal. Most of the pulmonary arterioles showed thickening and in some of them it was severe; it was represented in the arteriograms by poor filling of the peripheral fine vessels. 
Case 46. These abnormalities were well shown in our study of a man of 71 who had had coronary artery disease for many years. He had been admitted to hospital many times for left ventricular failure due to coronary artery disease. For four years before death he had been dyspnœic on effort. Necropsy confirmed the diagnosis of severe coronary sclerosis. Our measurement of his peripheral pulmonary arteriolar bed was $\mathbf{0 . 0 8}$. Many of the pulmonary arterioles showed severe subendothelial thickening (Fig. 4). There was a paucity of fine vascular markings in his post-mortem pulmonary arteriogram (Fig. 5).

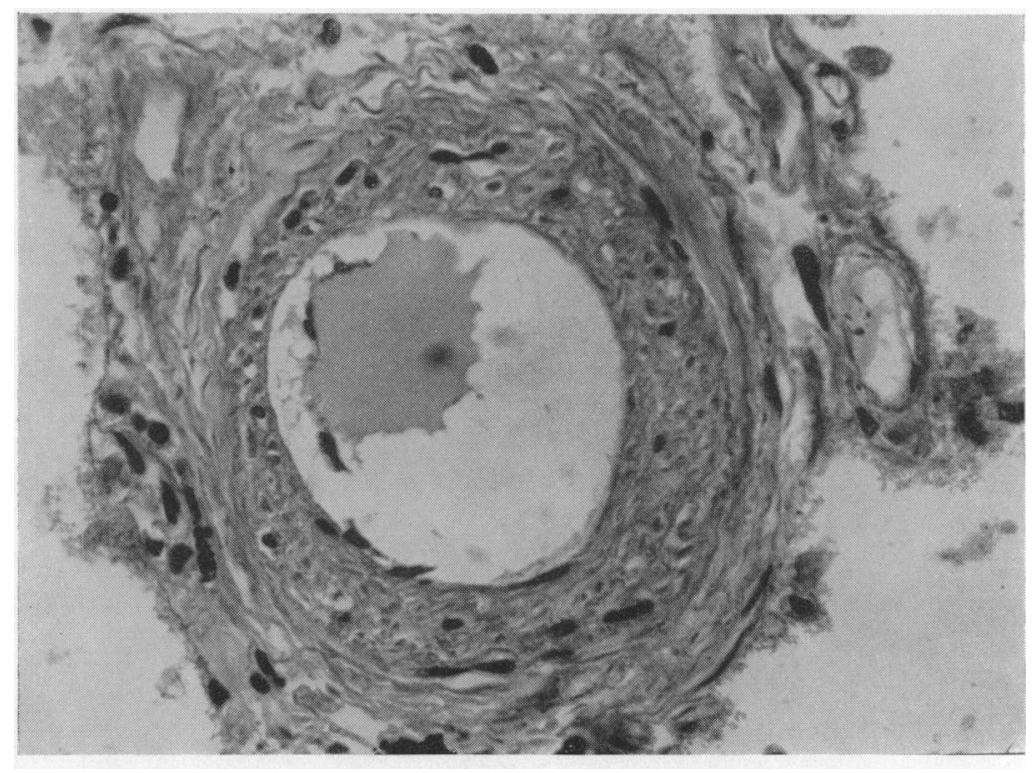

FIG. 4.-Case 46. A pulmonary arteriole from a man of 71 who had had many bouts of left ventricular failure. The subendothelial zone is grossly thickened. $(\times 500$.

\section{Discussion}

Temporary reductions in the pulmonary vascular bed can be caused by acute anoxia and some drugs (Motley et al., 1947; Borst et al., 1957). We have been interested in the anatomicalconstriction of the pulmonary arterioles found in some types of chronic heart disease and in the relationship of such arteriolar lesions to right heart stress and right ventricular hypertrophy. Pulmonary arteriolar lesions have been demonstrated in mitral stenosis (Short, 1957; Evans and Short, 1958). We have previously recorded arteriolar constriction in some cases of aortic stenosis (James et al., 1960). The present study has demonstrated similar lesions in patients with coronary artery disease who have had episodes of left ventricular failure. Such bouts of heart failure raise the pulmonary venous pressure (Lewis et al., 1953). We accordingly support the view that persistent or frequent elevation of pulmonary venous pressure resulting from left ventricular failure can lead to constriction of the pulmonary arterioles. In some cases the arteriolar lesions are severe enough to contribute to the development of right heart stress and right ventricular hypertrophy. 


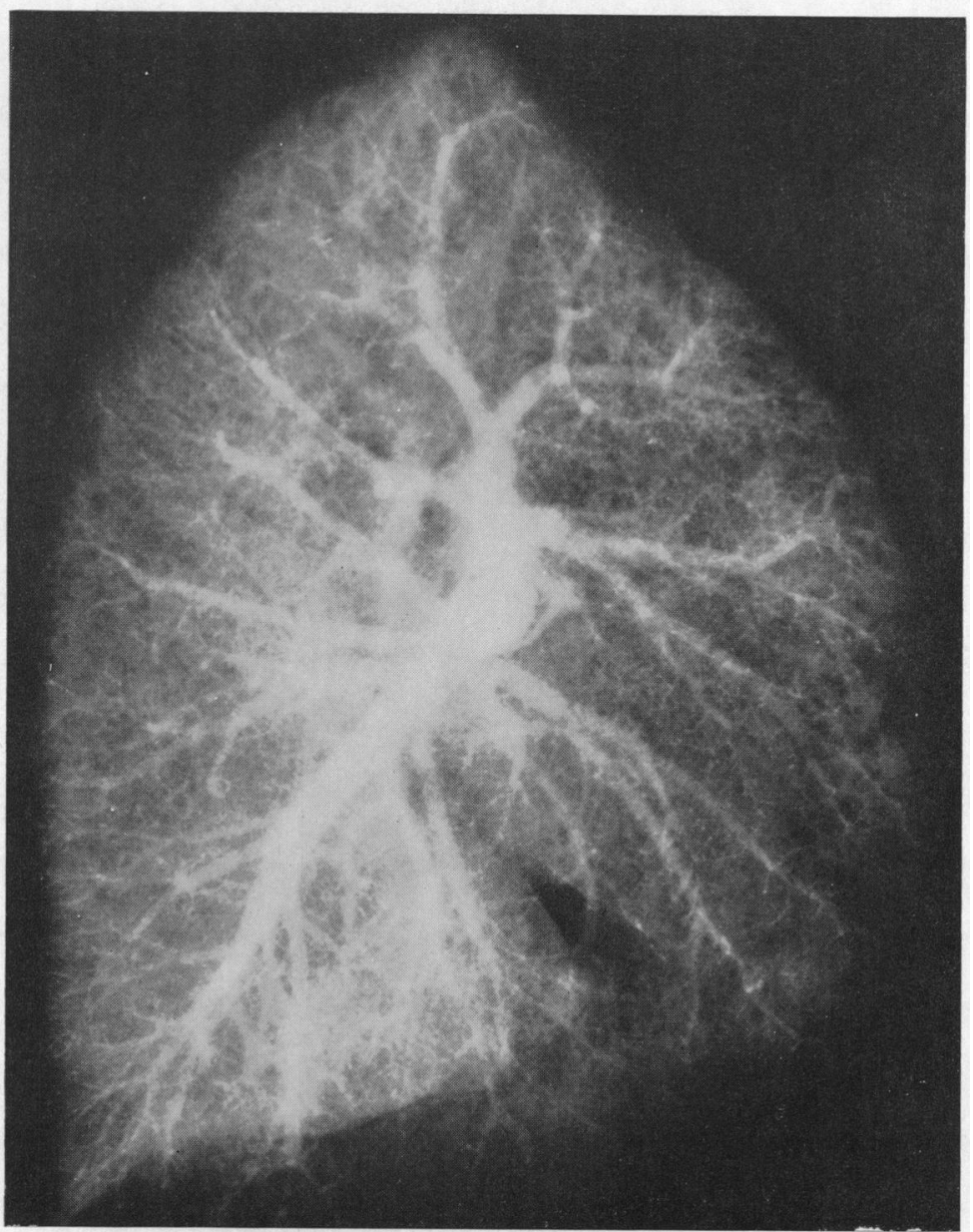

Fig. 5.-Case 46. In this post mortem pulmonary arteriogram the main vessels appear large and there is lack of filling of the peripheral small vessels. (About half size.)

\section{SUMMARY}

The pulmonary arterioles from fatal cases of coronary artery disease were studied by an injection method, by post mortem angiography, and histologically. Arteriolar constriction was demonstrated in the lungs of patients who had had episodes of left ventricular failure. It is suggested that the arteriolar lesions contribute to right heart stress by reducing the patency of the pulmonary arteriolar bed.

We are indebted to the pathologists of Sully, St. David's and Llandough hospitals for providing us with valuable specimens. We also thank Dr. Glyn Evans of the Cardiff Radiotherapy Centre. The photographs were taken by Mr. W. M. Travers of the Institute of Pathology of the Welsh National School of Medicine.

\section{REFERENCES}

Borst, H. G., Berglund, E., and McGregor, M. (1957). J. Clin. Invest., 36, 669.

Evans, W., and Short, D. S. (1958). Brit. Heart J., 20, 529.

James, W. R. L., Owen, G. M., and Thomas, A. J. (1960). Brit. Heart J., 22, 695.

Lewis, B. M., Houssay, H. E. J., Haynes, F. W., and Dexter, L. (1953). Circ. Res., 1, 312.

Motley, H. L., Cournaud, A., Werko, L., Himmelstein, A., and Dresdale, D. (1947). Amer. J. Physiol., $150,315$.

Short, D. S. (1957). Lancet, $2,12$. 